\title{
On-line Computing Facilities at Jodrell Bank
}

\section{J. G. DAVIES}

\section{Nuffield Radio Astronomy Laboratories, Jodrell Bank}

An on-line computer has been in use at Jodrell Bank since 1964, both controlling the Mk II radio telescope and performing data processing functions in connection with it. However this system is now being superseded, and the following describes the present state of development, including some facilities that are only now being constructed.

An Argus 400 computer is associated with each of the two main telescopes. Each has 12000 words of core store, the word length being 24-bits, and the time for obeying most functions is $12 \mu \mathrm{s}$. The 24-bit word length is well suited to the application since more than 16-bit accuracy is required in the coordinate transformation. This fact, and the very versatile organization of the Argus computer, makes the effective speed relatively higher when compared with faster machines of smaller word size. In addition a drum store of about 50,000 words capacity will be fitted to each machine, and CRT and graphical output devices are available. Each machine operates a permanent program, which performs a number of functions, including in the case of the Mk II telescope the control of the instrument itself. In addition, up to three "user" programs may be operated simultaneously; these may be on-line data collection, with links to the telescope control sections, or completely off-line background jobs. A wide selection of library routine, available to all programs, is permanently in the core store, and this makes the writing of programs a relatively simple matter.

Two methods are used for converting the outputs of radio receivers into digital form within the computer. In the first, the output power from the receiver is converted into a frequency of the order of $1 \mathrm{MHz}$. This is then counted in a binary counter, which is sampled at regular intervals by the computer. These time intervals may be synchronized with radio frequency switches at the front of the receiver, switching between two areas of sky, or between the sky and some comparison source of power. Thus the sum of all readings gives the mean power received, and the sum of the differences between alternate pairs gives the mean difference between the two responses. Alternatively, for example in pulsar observations, each successive sample can be added to the value for successive phases in the pulsar period, and then the mean profile of the pulse obtained.

The second method is used in interferometry and spectroscopy, where cross- and auto-correlation techniques are required. The signals are clipped and sampled in a binary fashion at a rate at least equal to twice the bandwidth. They are then multiplied in a series of one-bit multipliers (or in the case of the use as a spectrometer, the signal is multiplied by delayed versions of itself), and the result is summed in a series of counters. A system with 256 such channels capable of sampling at $10 \mathrm{MHz}$ is currently in use and one with 512 or 1024 channels sampling at up to $20 \mathrm{MHz}$ is planned.

The combination of telescope control and data processing in one computer is unusual. In several years' experience, no trouble has arisen from this, and it provides a very economical system. Since much of the program involved consists of routines required by the data processing sections, the core store used by exclusively control programs is less than 1000 words, and only about 5 per cent of the total time is used for this purpose. A considerable advantage is found in the ease with which user programs can inter-relate data collection and telescope control instructions.

\section{DISCUSSION}

C. L. STEPHENS: How long does your computer take to perform a 256-word Fourier transform, and to what accuracy is this done?

J. G. DAvies: The accuracy is of the order of 24-bit numbers-it's done in fixed-point-and the full 256 channel fast transform takes between 1 and 2 sec.

G. W. BothwelL: Could you give a brief explanatory breakdown of the way you use the 1000 words of core store that you quote are used for telescope control functions?

J. G. DAvies: The 1000 words is specifically for telescope control. In addition library routines are required to perform the trigonometry and for input and output functions, etc. In total these come to about 3500 words, all but 1000 of which are also available to time-shared programs concerned with data processing or other uses. The 1000 words refers to the actual servo loops, tests of satisfactory performance for safety purposes, and log keeping directly related to the telescope. 